\title{
Estudio sobre los retos de la educación presencial a nivel superior ante la contingencia sanitaria del COVID-19
}

\author{
Study on the challenges of fase-to-face education at a higher level in the fase of the health \\ contingency of COVID. 19
}

\author{
Irma Isabel de León-Vázquez, ${ }^{a}$, Delia Iliana Tapia-Castillo ${ }^{b}$, Daniel Vélez-Díaz ${ }^{c}$
}

\begin{abstract}
:
This document arises from the need to look for areas of opportunity to support students during a contingency period where face to face classes change to virtual classes and to observe how the human being is able to bear the weight of his fears and anxieties in a completely context new for him, where all paradigms were broken and spaces for innovation are created for students and teachers. It was necessary to immediately create a form in Google that would collect the information first hand and as soon as possible, since tha adaptation took place two weeks after the change began. Hence the to establish the media and the application to have a first impression of the upper level students during the COVID-19 pandemic.
\end{abstract}

Keywords:

Face-to-face education, virtual education, pandemic COVID-19

\section{Resumen:}

Este documento surge de la necesidad de buscar áreas de oportunidad para apoyar a los alumnos durante un periodo de contingencia, donde las clases presenciales cambian por clases virtuales y observar cómo el ser humano es capaz de ad aptarse a pesar de sus miedos y angustias a un contexto completamente nuevo para él, rompiendo todos los paradigmas y creando espacios de innovación para los alumnos y maestros. Fue necesario crear de manera inmediata un formulario en Google para recabar información en la primera semana y de primera mano lo antes posible, ya que la capacidad de adaptación de los alumnos se dio a las dos semanas de haber iniciado el cambio. De ahí la premura para establecer los medios de comunicación y aplicación para tener una primera impresión de los estudiantes del nivel superior durante la pandemia del COVID-19

\section{Palabras Clave:}

Educación presencial, educación virtual, pandemia COVID-19

\section{Introducción}

En este caso de estudio se describe el sentir inmediato de los alumnos a nivel superior en el municipio de Tlahuelilpan Hidalgo ante la pandemia (COVID-19) y la situación económica que los afecta con respecto a los recursos tecnológicos para poder adaptarse a una nueva rutina, sin bajar sus calificaciones o perder el semestre.
Estos estudiantes siempre han trabajado bajo la característica de clases presenciales y cuentan dentro de las instalaciones educativas con espacios para utilizar computadoras o internet, un gran porcentaje de los alumnos vienen de municipios diferentes y en muchas ocasiones no cuentan con computadoras o internet en casa.

Autor de Correspondencia, Universidad Autónoma del Estado de Hidalgo, Escuela Superior de Tlahuelilpan, ORCID: https://orcid.org/0000-0001-9197-0050,Email: irmalv@uaeh.edu.mx

b Universidad Autónoma del Estado de Hidalgo, Escuela Superior de Tlahuelilpan, ORCID: https://orcid.org/0000-0001-7522-543X, Email: itapia@uaeh.edu.mx

c Universidad Autónoma del Estado de Hidalgo, Escuela Superior de Tlahuelilpan, ORCID: https://orcid.org/0000-0001-6488-2960, Email: daniel@uaeh.edu.mx 
La investigación es importante porque permite observar a unas semanas de haber iniciado la pandemia (COVID.19) la reacción de los alumnos ante una forma diferente de clases y sus limitaciones tecnológicas, además servirá para que alumnos y docentes se esfuercen por crear o innovar nuevas estrategias de aprendizaje, siendo útil al buscar incorporar algunas alternativas de prevención educativa al término de este acontecimiento mundial y romper los paradigmas necesarios con el único fin de continuar con un trabajo de aula presencial a aula virtual, donde se debe trabajar con el autoaprendizaje.

El objetivo es describir las necesidades de los alumnos ante un cambio en la educación en periodo de contingencia a través de recopilar el sentir con encuestas digitales y videoconferencias, para crear el espacio de pensar en soluciones o alternativas que puedan ser trabajadas desde el aula como medida de prevención educativa para situaciones imprevistas.

Durante la investigación se tuvieron las restricciones y limitaciones como, por ejemplo: no se pudo contactar de forma inmediata a toda la comunidad estudiantil ya que se generó en la primera semana descontrol sobre lo que sucederá y cómo se llevará a cabo cualquier cambio.

\section{Exposición del Caso}

El municipio de Tlahuelilpan, Hidalgo se ha convertido en un lugar geográfico donde asisten una serie de estudiantes de diversas localidades a tomar clases desde los niveles básicos, como medio superior y superior. Todos están centrados en la modalidad presencial, una modalidad que permite al alumno en ocasiones asumir solo el rol de receptor, en espera que todos los saberes los comparta el docente, aún a pesar de estar consientes los docentes y alumnos de las competencias que deben ser desarrolladas.

Pero la llega de un periodo de contingencia ha obligado a trabajar a distancia de manera virtual en ocasiones sincrónica y otras asincrónica, rompiendo paradigmas no solo en el ámbito educativo también familiar.

Esto permitió observar de manera más marcada la desigualdad socioeconómica que los alumnos de esta región viven al no contar con recursos tecnológicos, ya que, al estar inscritos en una Universidad Pública, este tipo de herramientas como son las computadoras o el internet lo tienen en las instalaciones de la escuela.

\section{Conocimiento previo}

Desde 2017 se han presentado diversas trabajo que manifiestan la necesidad del uso de tecnología en la educación, tal es el caso del trabajo "La tutorial digital: un reto generacional o una deficiencia en equipamiento tecnológico" donde en el Segundo apartado llamado "Equipamiento tecnológico Vs Acceso a Internet" describe lo que ha sucedido en México con respecto al equipamiento tecnológico a las instituciones educativas y presenta los datos del Censo del 2013 donde hace referencia a la diferencia que hay entre los estudiantes de escuelas públicas y privadas con respecto al acceso a equipo.

También hace referencia a que a pesar de hablar de escuelas de calidad la verdad es que solo el $46 \%$ cuenta con conexión de internet.

Además, se indica que se puede tener alu mnos que tienen la tecnología en la mano y que los profesores se han preparado con cursos de TIC y pedagogía y las instituciones cuentas con plataformas educativas y que solo es cuestión de estar preparados para el cambio.

En el artículo "La cultura digital en la escuela pública" hace referencia que podemos estar conectados pero que la tecnología sin pedagogía no es suficiente.

En un documento online que habla de "los retos escuela en casa", hace referencia a que, debido a la contingencia COVID-19, los padres de familia, los docentes y los alumnos buscan adaptarse a una nueva rutina de trabajo lo que implica aprender en línea. Creando una controversia con respecto a las horas que debe dedicar el alumno a estudiar en clase.

Por ello es necesario recolectar información para poder explicar las dudas e inquietudes que se tiene con respecto a los cambios que rompen paradigmas para posteriormente ajustar las piezas del proceso socioeducativo.

\section{Metodología de diseño o de estudio}

La metodología que se aplicó fue cuantitativa y cualitativa una combinación de estudio mixto que permite conocer números de las necesidades, pero también aspectos que solo pueden ser comprendidos desde la postura de quién está viviendo la situación.

Para el desarrollo del trabajo, en esta situación de contingencia, fue necesario aplicar un formulario de Google para poder recabar la información, contando con un total de 23 preguntas: 17 de opción múltiple y 6 preguntas abiertas, que fueron enviadas por correo electrónico. Así mismo, se realizaron entrevistas a través de videoconferencias a los estudiantes lo que permitió conocer mejor el contexto de las necesidades que se presentan. Este estudio se realizó en la primera semana del periodo de contingencia y se dio seguimiento dos semanas más, con una participación de 163 estudiantes. 


\section{V.Resultados}

Los alumnos que contestaron el formulario fueron en un $60 \%$ del área académica de enfermería y un $40 \%$ de administración. Teniendo mayor participación los alumnos de primer, tercer, sexto y quinto semestre.

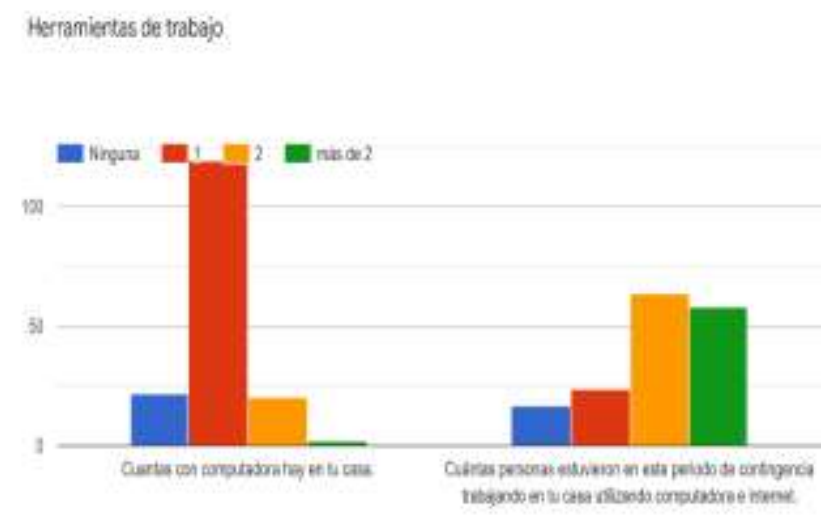

Con respecto a número de computadoras con las que cuentan en casa 119 alumnos solo tienen una computadora y las ocupan entre dos o más personas.

Con qué platálorma te hubiera gustads trabajar en este periodo de contingencia Haspouta

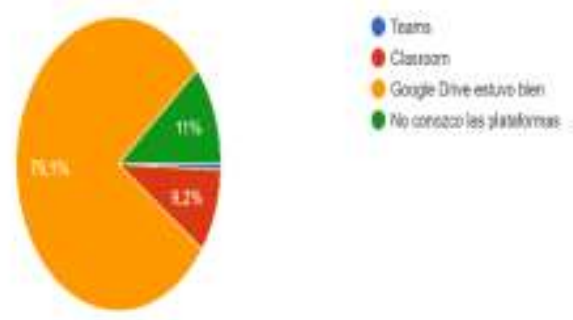

Al parecer los alumnos se adaptaron a trabajar en Google Drive y Classroom, sin embargo, un $11 \%$ desconocía las plataformas para trabajar de manera virtual.

Cuàras horns al da estableciste para realizar tus inabajos escolares? THesenatian
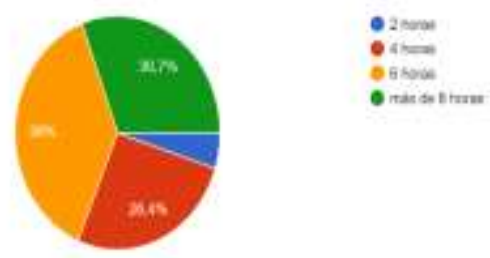

En lo que corresponde a las horas que se establecieron para realizar trabajos escolares el $38 \%$ ocu po 6 horas y el $30 \%$ más de 8 horas diarias, de éstos últimos el $71 \%$ indica que se debió a que fue de momento demasiada información. El 32\% de los alumnos no estableció horanios de trabajo debido a la carga de trabajo emitida.

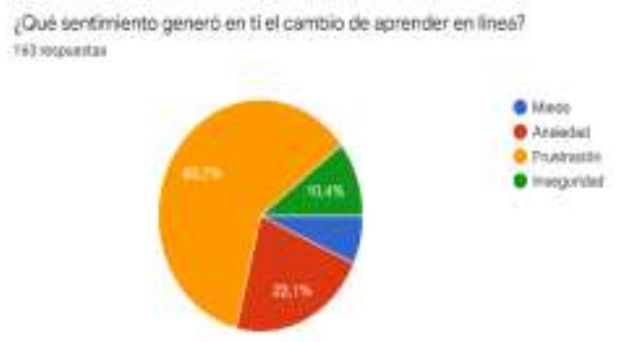

Con respecto al sentimiento que tuvieron los alumnos al trabajar en línea fue frustración y ansiedad. Originado por que las instrucciones dadas por los maestros no fueron claras.

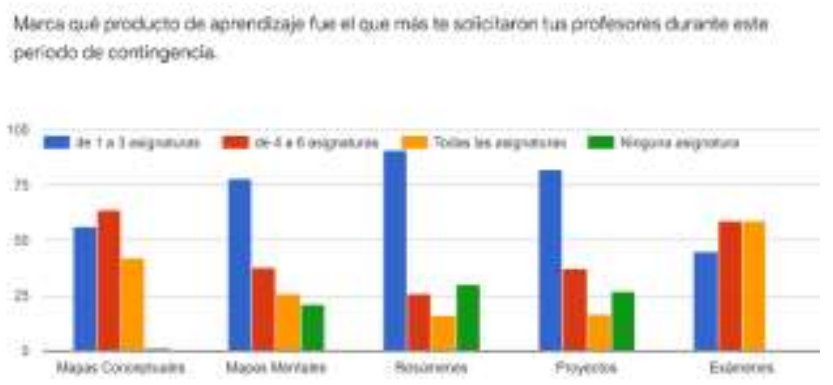

Los profesores se enfocaron a solicitar diversos productos académicos con el fin de encontrar evidencias para colocar una calificación entre los que destacan los resúmenes, los mapas y los proyectos.
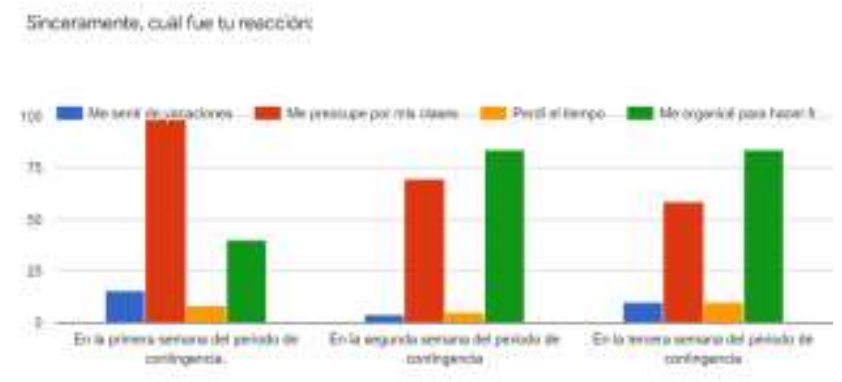

Se puede observarque en la primera semana los alumnos se preocuparon por sus clases, para la segunda semana comenzaron a organizarse.

\section{Discusión}

De acuerdo a lo revisado a lo largo del formulario, se observa que fue necesario establecer estrategias para que todos los integrantes de la familia pudieran hacer uso de la computadora y del internet, estableciendo horarios 0 turnos de trabajo para realizar las tareas empezando desde muy temprano y algunos trabajos los realizaban en celulares. 
También se les pregunto sobre cómo reaccionarían ante otro periodo de contingencia a lo que respondientes que buscarían organizarse de mejor manera para estar preparados, tratarían de adaptarse en menos tiempo y con esta experiencia sientes que ya sabrían cómo actuar. Aunque algunos alumnos sufren de an siedad y esta forma de trabajo los inquieta más, ocasionando problemasen su aprendizaje al tener una cantidad inmensa de dudas y consideran que sería necesario que las escuelas estuvieran preparadas para este tipo de situaciones.

\section{Conclusiones}

Toda esta situación ha generado diversas formas de pensar con sus pros y contras, ya que se aprendió a trabajar desde casa, a organizar tiempos, a ser autodidactas, a cuidar la salud. También generó miedos, frustraciones, inconformidad, angustia. Hace reflexionar que tanto los alumnos, como las instituciones deben no solo dar clases presenciales, sino también durante el periodo de clases trabajar bajo esta forma que se ha aprendido. De esta manera los alumnos tendrían que aprender a leer mejor las instrucciones para saber lo que se le pide y los profesores tendrían la oportunidad de desarrollar un diseño instruccional que es fundamental en la educación a distancia. Cada materia debería en un curso normal establecer algunas actividades en línea para instaurar los parámetros en los que se puede crear e innovar en la educación.

Los alumnos estarían preparados para realizar trabajos desde su casa en línea, evitando el miedo a lo nuevo y con ello crear fuentes de trabajo que se pueden realizar desde cualquier lugar del mundo, siempre y cuando se haya aceptado a esta forma de comunicación.

\section{Referencias}

Cajal, M. (19 de diciembre de 2014). Estrategias de Marketing Digita 1. Obtenido de Millennials, Generación "X" Cómo se compor-tan online. https://www.mabelcajal.com/2014/12/millennials-generacion-x-babyboomers-como-se-comportan-online.html/

Clarín. (7 de Julio de 2017). Tecnología y Trabajo - Generaciones. Obtenido de https://www.clarin.com/entremujeres/carre-ra-ydinero/baby-boomers-generacion-millennials-centennials-generacionperteneces_0_ByLAxzpEW.html

De León, I; Tapia, D; Vélez, Daniel (2019). $8^{\circ}$ Encuentro Nacional de Tutorías. Innovación para la permanencia con equidad educativa. "La tutoría digital: un reto generacional o una deficiencia en equipamiento tecnológico" pag. 1233-1236. UASLP-ANUIES. México. Obtenido de https://ua slpedu-

my.sharepoint.com/personal/jorge_nunez_uaslp_mx/_layouts/15/onedr ive.aspx $? \mathrm{id}=\% 2$ Fpersona $1 \% 2 \mathrm{Fjorge} \% 5$ Fnunez $\% 5$ Fua slp $\% 5 \mathrm{Fm} \times 2 \mathrm{FD}$ ocuments $\% 2$ FInnovaci\%C3\%B3n $\% 20$ para $\% 201 \mathrm{a} \% 20$ permanencia $\% 2$ 0 con $\% 20$ equidad $\% 20$ educativa $\% 2$ Epdf $\&$ parent=\%2Fpersona $1 \% 2$ Fjorg

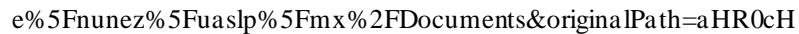
M6Ly91YXNscGVkdS1 teS5zaGFyZXBvaW50LmNvbS86YjovZy9w ZXJzb25hbC9qb3JnZV9udW5le191YXNscF9teC9FVUt3ZGt1anRfZF
B1RFFNMFkxdDV6c0I0UnhjeTltb1JUWnA0SIRiZV9OSmhnP3J0a W1IPS1TZE03U0h1MTBn

FayerWayer. (2014). Censo educativo: la tarea del acceso a las TIC en México. Obtenido de https://www.fayerwayer.com/2014/10/censoeducativo-acceso-tic/

Graduate XXI \& Futuro Educativo.(2018). BID. Obtenido de El Uber de la educación: los tutores digitales: http://www.gradua-texxi.org/el-uberde-la-educacion-los-tutores-digitales/

López-Gil, M. M., \& Bernal-Bravo, C. (22 de Julio de 2015). UAEM Redalyc. Obtenido de La cultura digital en la escuela pública: http://www.redalyc.org/jatsRepo/274/27446519010/html/index.html

Prieto, Heriberto (2020). Educación Futura. "Ante la contingencia sanitaria, los aprendizajes con tecnologías digitales". Obtenido de http://www.educacionfutura.org/48646-2/

SEP. (2013). Secretaria de Educación Pública. Obtenido de Censo de Escuelas, Maestros y Alumnos: http://www.censo.sep.gob.mx/UAEH. (2016).

UNICEF (2020) COVID-19: UNICEF llama a la comunidad educativa ante medidas de contingencia. Obtenido de https://www.unicef.org/mexico/comunicados-prensa/covid-19-unicefllama-la-continuidad-educativa-ante-medidas-de-contingencia

UNIVERSIDAD AUTÓNOMA DEL ESTADO DE HIDALGO. Obtenido de Prontuario del tutor: https://www.uaeh.edu.mx/tutorías/docs/prontuario_del_tutor__ver_4_1 -.pdf 